discharge newborn thrived well but had a severe motor delay. $\mathrm{He}$ was referred to our institution at the age of 3 months with severe hypotonia, hypermobile joints, extremities in a frog-like position, redundant skin, no head control, bell-shaped thorax with pectus excavatum, mild thoracolumbar kyphosis, spina bifida occulta, umbilical hernia, undescended testicles, and talipes calcaneovalgus. Social contact was normal. Metabolic workup and gene analyses for spinal muscular atrophy, myotonic dystrophy type 1 and Prader-Willi syndrome gave normal results. Brain MRI was normal.

Electromyoneurography showed myopathy. Muscle biopsy revealed abnormal mitochondria and decreased activities of all respiratory chain complexes.

Mitochondriopathy was suspected and ,mitochondrial-cocktail' therapy was started. At two years of age, the patient was severely hypotonic, with myopathic face, hyperelastic joints and skin, easy bruising, thoracolumbar kyphoscoliosis, and normal mental development. He started to walk at the age of 3.5 years. At the age of 5 years, he developed severe osteoporosis and kyphoscoliosis progressed. Whole exome sequencing (WES) revealed homozygous mutation c.362dupC (p.Glu122fs) in the FKBP14 gene.

Conclusion Collagen myopathies should be in the differential diagnosis of floppy infant syndrome. Skin hyperextensibility and improvement of motor function with age may help to distinguish kEDS from collagen VI-related myopathies. Diagnosis can be confirmed by single gene or multigene panel testing. In a diagnostic dilemma, as in this case, in which some findings pointed to the diagnosis of mitochondrial disease, WES could be the best diagnostic approach. Accurate diagnosis ensures optimal patient management, complications insight, and appropriate genetic counselling.

\section{KABUKI SYNDROME: REVIEW OF THE CLINICAL FEATURES AND DIAGNOSTIC FINDINGS IN FOUR CROATIAN PATIENTS}

Sanda Huljev Frković*, Tin Kranjec, Mia Šalamon Janečić, Mario Ćuk, Marijan Frković, Kristina Crkvenac Gornik, Marija Jelušić. Department of Pediatrics, University Hospital Centre Zagreb, University of Zagreb School of Medicine

\subsection{6/archdischild-2021-europaediatrics.87}

Goal Kabuki syndrome (KS) is a rare malformation syndrome caused by mutations in KMT2D and KDM6A genes. Besides the cardinal manifestations, including distinctive facial appearance, mild-to-moderate intellectual disability, dermatoglyphic abnormalities such as persistence of fetal fingertip pads, skeletal anomalies and postnatal growth retardation, a wide spectrum of other anomalies is documented so far. We present clinical features and diagnostic findings in our four patients.

Methods During the 2016-2019 period, four of our patients were diagnosed with KS based on clinical observation and gene-targeted testing or comprehensive genomic testing (exome sequencing).

Results Three of our patients were males, one aged 17 and two aged 3 years, and one female aged 16 years at the time of diagnosis. Mutation of the KMT2D gene was identified in two patients, mutation KDM6A in one patient, while molecular analysis of one male patient is still in progress.

In addition to the five cardinal findings, one male patient had cleft palate, one had aortal coarctation with bicuspid aortic valve and one had multiple severe respiratory infections. Although susceptibility to autoimmune disorders is a known feature in these patients, our female patient with KMT2D gene mutation was recognized as the first KS patient with systemic lupus erythematosus.

Conclusion KS is a rare disorder with highly variable clinical features making the diagnosis difficult. Consequently, a close collaboration between pediatric subspecialists, clinical geneticists and molecular biologists is essential in the terms of clinical recognition and genetic confirmation of this syndrome.

\section{LOWE SYNDROME - OLD AND NEW EVIDENCE OF SECONDARY MITOCHONDRIAL DYSFUNCTION}

Katja Dumic Kubat*, Darko Anticevic, Jelena Petrinovic-Doresic, Tamara Zigman, Kamelija Zarković, Matea Melša, Oliver Vugrek. Department of Pediatric Endocrinology and Diabetes, University Hospital Centre Zagreb, University of Zagreb, Medical School

\subsection{6/archdischild-2021-europaediatrics.88}

The oculocerebrorenal syndrome of Lowe (LS) is a rare, progressive, multisystemic X-linked disorder caused by mutations in OCRL gene. Patients classically present with ocular abnormalities including bilateral congenital cataracts and glaucoma, intellectual delay, severe generalized hypotonia with absent tendon reflexes, and proximal renal tubular dysfunction. Congenital bilateral cataracts and hypotonia are present at birth in almost all patients, while other classical symptoms develop gradually with variable severity. Consequently, differential diagnosis in infant period in these patients can be broad including other rare metabolic and neurologic disorders.

Herein we present a 4.5 year old boy with Lowe syndrome caused by novel mutation of OCRL gene (c.643C>T/p. Gln $\left.215^{*}\right)$ initially diagnosed as having mitochondriopathy due to alteration of mitochondria on electron microscopic examination in different tissues and decreased values of mitochondrial energy metabolism measurements in muscle. No pathogenic mutations in mitochondrial DNA were found on whole exome sequencing.

This patient recall historical hypothesis of secondary mitochondrial dysfunction in Lowe syndrome, that may be caused/ intensified some of disease symptoms.

\section{FIVE-YEAR UPDATE FOR THE PHASE III VORETIGENE NEPARVOVEC STUDY IN BIALLELIC RPE65 MUTATION- ASSOCIATED INHERITED RETINAL DISEASE}

Bart P Leroy*, Stephen R Russell, Jean Bennett, Katherine A High, Arlene V Drack, ZiFan Yu, Amy Tillman, Daniel Chung, Kathleen Z Reape, Albert M Maguire. Department of Ophthalmology and Center for Medical Genetics Ghent, Ghent University and Ghent University Hospital, Ghent, Belgium; Children's Hospital of Philadelphia, Philadelphia, PA

\subsection{6/archdischild-2021-europaediatrics.89}

Goal To determine whether ambulatory navigation, light sensitivity, and visual field (VF) improvements 1 year after voretigene neparvovec $(\mathrm{VN})$ administration in patients with biallelic RPE65 mutation-associated inherited retinal dystrophy (IRD) are maintained at 5 years and review safety outcomes over the entire period.

Methods This is an open label, randomized, controlled Phase III trial performed at 2 sites in the United States. Patients were randomized to either original intervention (OI: bilateral subretinal VN at baseline; $\mathrm{n}=20$ ) or delayed intervention (DI: $\mathrm{VN}$ after 1 year; $\mathrm{n}=9$ ). The primary endpoint was bilateral 
performance on the Multi-Luminance Mobility Test (MLMT) at 7 standard light levels as measured by a change in score. Additional endpoints were full-field light sensitivity threshold (FST) testing, visual acuity (VA), and Goldmann kinetic VF (GVF), each averaged over both eyes. Safety outcomes included adverse event reporting, laboratory testing, and changes in physical and ophthalmic examinations.

Results For OI patients at Year $5(n=18)$ and DI patients at Year $4(\mathrm{n}=8)$, the mean (standard deviation) MLMT bilateral light level score change was 1.6 (1.1) and 2.4 (1.5) levels, respectively, compared with baseline. Subsequent to the 1-year outcomes, a change of 1 light level occurred in 6 patients (none were below pre-treatment performance) and no change in the remaining $20(\mathrm{~N}=26)$. Mean change in white light FST in $\log 10$ (cd.s $/ \mathrm{m} 2$ ) averaged over both eyes was -2.02 (1.45) $\log 10$ at Year 5 for OI patients $(n=17)$ and -2.58 (1.04) $\log 10$ at Year 4 for DI patients $(n=8)$. Mean change in VA (Holladay Scale) averaged over both eyes (logMAR was -0.00 $(0.64)$ at Year 5 for OI patients $(n=18)$ and $-0.06(0.26)$ at Year 4 for DI patients $(n=8)$. Mean change in GVF III4e sum total degrees averaged over both eyes was 166.6 (208.7) at Year 5 for OI patients $(n=15)$ and $178.8(241.9)$ at Year 4 for DI patients $(n=8)$. Five years after treatment, the safety profile $(\mathrm{N}=29)$ was consistent with vitrectomy and subretinal injection procedure with 2 reports of cataract, 1 of ptosis, and 1 new report of retinal detachment since the last update. No deleterious immune responses were reported.

Conclusion Improvements in ambulatory navigation, light sensitivity, and VF are maintained for at least 5 years after $\mathrm{VN}$ administration in most OI patients.

Improvements in DI patients were consistent with those observed in OI patients. The safety profile of $\mathrm{VN}$ is consistent with the administration procedure.

\section{\begin{tabular}{l|l}
90 & THE FIRST CLINICAL CASE OF RARE FORM OF FOCAL
\end{tabular} EPILEPSY CAUSED BY THE NOVEL MUTATION IN THE NPRL3 GENE IN RUSSIAN FEDERATION AND KAZAKHSTAN}

Savostyanov Kirill, Nauryzbayeva Alisa, Globa Oksana, Pushkov Alexander*, Kuzenkova Lyudmila, Kondakova Olga, Pakhomov Alexander, Muraveva Lyubov, Fisenko Andrey, Altynshash Jaxybayeva. National Medical Research Center for Children's Health of the Ministry of Health of the Russian Federation

\subsection{6/archdischild-2021-europaediatrics.90}

Objective Mutations in the NPRL3 gene (OMIM 600928) are described predominantly in patients with autosomal dominant focal epilepsy according to the HGMD database.

Methods We want to introduce the clinical case of the female patient, 2 years old, with focal epilepsy from healthy parents. She was born full-term from the 1st pregnancy by caesarian delivery. The cerebellum hypoplasia was suspected during ultrasound and MRI diagnostic at 17 th week of gestation.

Weight at birth - $3.780 \mathrm{~kg}, 7 / 8$ on Apgar scale. Neurosonography at birth has shown left-sided ventriculomegaly. First recurrent afebrile tonic extensor epileptic spasms with head and eyes turns to the left side were noted at the age of 2 months. Eyelid and tongue myoclonias were revealed as well. Later the frequency of seizures has increased to 150 per day. The video electroencephalogram (VEEG) showed sharp, spike waves with partial origin at left temporal region, while clonic seizures in the left arm were recorded. The brain MRI has shown focal cortical dysplasia in the left insular region. Signs of intellectual impairment and behavioural disturbances were revealed during the neurological examination at the age of

24 months. The patient could not walk without support. We have performed full exome sequencing for our patient to identify the molecular genetic causes of the disease after medical genetic counseling.

Results The nucleotide variant c.481C $>\mathrm{T}$ which leads to stop codon p.Q161* in heterozygous state was revealed in exon 5 of the NPRL3 gene. This variant was not described in gno$\mathrm{mAD}$ and HGMD databases and was considered as pathogenic according to ACMG criteria. An interesting fact is that the most frequent pathogenic variants in the NPRL3 gene (among the 21 described variants in the HGMD) are nonsense mutations and frameshift deletions.

Conclusion This paper describes the first clinical case of rare form of focal epilepsy caused by the novel mutation in the NPRL3 gene in Russian Federation and Kazakhstan.

\section{FABRY'S DISEASE WITH MINIMAL MANIFESTATIONS IN GIRLS (CLINICAL CASES)}

Gadzhikerim Gadzhikerimov*, Olga Gumeniuk. Saratov State Medical University

\subsection{6/archdischild-2021-europaediatrics.91}

Fabry disease (Anderson-Fabry disease) is a rare inherited lysosomal multisystem disease with X-linked inheritance due to the deficiency of lysosomal enzyme $\alpha$-galactosidase $\mathrm{A}$ and leads to accumulation of sphingolipids (globoside, globotriaosylceramide) in the walls of blood vessels in all organs.

Clinical cases of low-symptomatic Fabry's disease in 2 girls (9 and 10 y.o.) with positive family history of the underlying disease are described.

Girl 9 y.o. is granddaughter and girl 10 y.o. is daughter of a male patient with Fabry's disease. Girls had complaints of reduced sweating and febrile acroparastesia only.

Physical and sexual development according to age, the skin was clean.

Echocardiography, abdominal USE, pulmonary function test, cornea and fundus, ear, nose and throat examination were normal. Urine examination showed $1+$ proteinuria both girls have. Glomerular filtration rates were normal. The lyso-Gb3 and galactosidase A levels analyzed in dried blood spots (DBS) by tandem mass spectrometry and molecular genetic analysis was performed. An increase lyso-Gb3 concentration and a change in the nucleotide sequence c. $983 \mathrm{G}>\mathrm{C}$, leading to the replacement of p.G328A, described in the international database on HGMD mutations (CM 930337) in the hemizygous state has been identified.

Conclusion These cases confirm that it is very important that the pediatri $\square$ ians become aware of the importance of genealogical anamnesis findings and Clinical features of Fabry's disease, so they can participate in the identification of unrecognized patients.

\section{TYPICAL PROBLEMS OF PARENTS OF CHILDREN WITH CLEFT LIP AND PALATE}

Evgenija Shatova*. FGAOU V.O. First Moscow State Medical University named after I.I. THEIR. Sechenov, Ministry of Health of the Russian Federation

10.1136/archdischild-2021-europaediatrics.92 